\title{
Clinical Pharmacist- A Need for the Society
}

\author{
Fatima Tahniyath
}

Quality Manager, Aster Prime Hospital, Ameerpet, Clinical Pharmacist-An Asset, Hyderabad, Telangana, INDIA

\begin{abstract}
Introduction: "Opportunities are growing for clinical pharmacists," says Dr. Joe Calomo, assistant dean of experiential education and professional affairs at the Massachusetts College of Pharmacy and Health Sciences in Boston. "More hospital administrators are beginning to see the value of having a clinical pharmacist on staff, because it can save costs and improve patient outcomes." Background: The clinical pharmacy has spread excessively due to its professional services throughout the past few years. Now the clinical pharmacy profession has been recognized as an eminent and important profession in the multidisciplinary setup of health care as they became a crucial element of healthcare team to promote patient care by interacting with physician and patient. They can assist in therapeutic decision making and the preparation of guidelines for antibiotic usage by assessing cost effectiveness. Conclusion: The clinical pharmacists are granted patient care privilege by interacting with physicians or health systems that allow them to perform medication decision making functions as part of the patient's health care team. Thus, Clinical Pharmacists are an asset for health care team and patients.
\end{abstract}

Key words: Pharmacist interventions, clinical pharmacist, patient counseling, healthcare provider, multidisciplinary.

\section{INTRODUCTION}

With more pharmacists growing weary of day-in and day-out pill-dispensing duties, clinical pharmacy is attracting druggists who want to play a vital role on the healthcare team.

The clinical pharmacy has spread excessively due to its professional services throughout the past few years. Now the clinical pharmacy profession has been recognized as an eminent and important profession in the multidisciplinary setup of health care as they became a crucial element of healthcare team to promote patient care by interacting with physician and patient.

As clinical pharmacists have precise knowledge about therapeutics and regular interaction with prescribers help them to bridge the gap between patients and physicians. The association of clinical pharmacist and clinician can provide a strong base for quality assured patient care.

The presence of a clinical pharmacist in the ward is to be regarded as a revolutionary feature of developments in the pharmacy field.

\section{Activities of a Clinical Pharmacist}

Clinical pharmacists participate in ward rounds with physicians and provide their suggestions or recommendations needed for patient's well-being. The physicians prescribe medicines and the dutiful pharmacist's checks the prescription to ensure rational use of drugs by marking the presence of an indication for the drug, if it is in the right drug/dose/duration/dosage/time, etc. If there is any variance from these, they inform the prescriber, document and make appropriate interventions. Complimentary agreement between the physicians regarding the impact of the intervention indicates that the pharmacists' intervention had a confirmed outcome on therapy.

Clinical pharmacists contribute their efficiencies in medication review, identification of drug related problems, therapeutic recommendations and
DOI: 10.5530ijopp.10.1.12

Address for correspondence: Dr. Fatima Tahniyath, Pharm.D Quality Manager, Aster Prime Hospital, Ameerpet, Clinical Pharmacist-An Asset, Hyderabad, Telangana, INDIA. Phone no: 9948219786 E-mail: ftahniyath@gmail.com 
promotion of medication compliance. They obtain medical and medication history, check medication errors including prescription, dispensing and administration errors, identify drug interactions, monitor adverse drug reactions (ADR), suggest individualization of dosage regimen, provide patient counseling, etc. If the patient has an adverse reaction to a drug, then it is the clinical pharmacist's responsibility to notify the doctor and suggest a better treatment. Clinical pharmacists also monitor dosages to make sure patients are getting enough -- but not too much -- of the drug therapy. In addition to this, clinical pharmacists do drug dilutions, extemporaneous preparations, dose calculations, etc.

Participation of a clinical pharmacist in ward/ICU rounds and clinical discussions helps to identify, prevent or reduce drug interactions and ADR. In addition to this, a clinical pharmacist suggests a cost-effective patient compliant therapy and in creating a database for each drug as well as in clinical trials.

Pharmacists have an up-to-date knowledge with the changing world of medicine and they can contribute effectively for clinical research projects or other ongoing research programs Clinical pharmacy services develop and maintain a clinical practice with a patient care service, cooperating with medical and nursing personnel to optimize the pharmacotherapeutic aspects of patient care, ${ }^{2}$ which provides optimal selection of pharmacologic agents, and design of a regimen to maximize therapeutic benefits and minimize toxicity. Clinical pharmacists provide daily follow-up on the clinical effects of the regimen with adjustments as necessary to achieve desired outcomes.

\section{Services of a Clinical Pharmacist in Hospital Expanse}

Clinical pharmacists conduct a complete patient interview on medical history, social and family history, and history of allergy, use of OTC drugs, dietary supplements and alternative systems of medicine. Clinical pharmacists perform review of drug therapy which helps to recognize and utilize relevant clinical and lab data to identify and resolve drug related problems such as duplication of therapy, drug-drug and drug-food interactions, contraindications, inappropriate dosage in terms of frequency \& strength, lack of basic lab monitoring requirements, potential ADRs, inappropriate drug selection, drug therapy without indication or no drug order for an indication, non-adherence to medications and availability of cost effective alternatives. They can assist in therapeutic decision making and the preparation of guidelines for antibiotic usage by assessing cost effectiveness.

Provision of unbiased, up-to-date information on any aspect of drug use is another major responsibility of a clinical Pharmacist. They are capable of providing information on strength, availability of drug formulations, brand and cost.

They provide information regarding dosing of drugs in patients with renal or hepatic impairment function. In patients with renal impairment, clinical pharmacist should emphasize on drug dosing adjustments. An increase in morbidity and mortality as well as therapeutic costs will be the consequences of failure in doing dosage adjustment where it is highly recommended to estimate creatinine clearance prior to drug ordering and use reliable dosing guideline. The adjustment of dose can be done by interval extension or dose reduction. Estimates of renal function are helpful in identifying patients who may need shorter dose intervals (high clearance) or may be adequately treated with smaller dose amounts or longer dose intervals (low clearance).

In case of Hepatic impairment Patients who underwent organ transplantation are advised to take multiple drugs and adherence to these medications is important to get around graft rejection and medical costs. A clinical pharmacist can provide help to prevent the progression of ESRD by educating and motivating the risk group patients for regular checkup and explaining them about medication adherence, counseling them about lifestyle modification.

Since a clinical pharmacists have thorough knowledge about the drugs they can easily identify and notify as look-alike and sound alike drugs. They can actively participate in therapeutic drug monitoring, medical camps and patient awareness programs on medication usage.

Therapeutic Drug Monitoring is an important part of the Clinical Pharmacists activity to monitor the patient, be it a simple drug as Paracetamol, a patient's favorite pill which has a psychological impact too that without it he will not be able to work even though it is not required, there are several such examples which are to be taken into consideration during ward rounds.

Clinical pharmacists ensure that reconstitution, dilution, stability, storage, compatibility and administration of drugs are to be carried out appropriately. They facilitate conversion of parenteral to oral dosage forms when indicated. Provision of alert cards for selected patients who suffer from ADR or take medications that require special warning/caution (epilepsy, cardiac problem, drug 
allergy or taking insulin, warfarin, aspirin, etc.) is another major responsibility.

They have an active participation in physician rounds in all the departments of a hospital. They provide patient counseling on usage of medicines, the use of devices like inhaler, insulin pen, eye drops, nasal sprays, administration of insulin injection, dietary restrictions, lifestyle modifications, importance of regular checkup etc. The clinical pharmacist education sessions and medication adherence programs (pill box, use of diary logs and follow up calls) for patients proved beneficial in reducing mean FBS and $\mathrm{HbA1c}$ significantly. ${ }^{3}$

Clinical pharmacists in pediatrics and neonatology can help in dose calculation and dosage form modification. The increased need for calculations and dilutions of pediatric medicines, and a requirement to adjust dose of an individual patient based on age, gestational age, weight and surface area, provide a greater opportunity for a clinical pharmacist in medication management process. They can help to prepare pediatric/neonatal formulary; the need of immunization can be well explained, mothers can be well educated about the importance of breast feeding and can be counseled regarding medications. As some parents use the same medications if the baby has same complains next time which should be avoided.

Clinical pharmacists in the Department of Neurology can identify risk group of patients (bleeding), potential drug interactions and reduce adverse effects. They monitor the INR range of patients taking warfarin, and can seek proper advice from the physician regarding dose adjustment in warfarin patients, provide counseling about dietary changes, need for INR monitoring, adverse effects, its management, etc.

Clinical pharmacist in oncology department calculates the body surface area (BSA) for the patients who are due for the next chemotherapy based on their height and weight and titrate their doses for the next session.

Clinical pharmacy services are not limited to the above mentioned activities. Clinical pharmacists can implement ever-expanding roles like therapeutic drug monitoring and managing of patient's pharmacotherapy which significantly improve patient care. The features of interactions occurring between clinical pharmacists and physicians influence the teamwork between pharmacists and physicians to provide better patient care. Clinical pharmacist can find a solution for a number of queries which includes information of drug profile, indication or dosage, adverse drug events, patient management, drug interactions, drug use in pregnancy and lactation, poisons and information regarding storage of drugs, etc.

They work with patients and other health professionals, to support and assist patients to make lifestyle changes to improve outcome of the patient.

\section{CONCLUSION}

The clinical pharmacists are granted patient care privilege by interacting with physicians or health systems that allow them to perform medication decision making functions as part of the patient's health care team to promote rational use of drugs for patient's safety and to improve patient care, level of attention and disease management result in control of risk factors and decline in health care costs.

Thus, Clinical Pharmacists are an asset for health care team and patients.

\section{CONFLICTS OF INTEREST}

No Conflicts of Interest to declare.

\section{ACKNOWLEDGEMENT}

I would like to acknowledge Dr Syed Abdul Azeez Basha, Principal, Deccan School of Pharmacy for his eternal support and Guidance.

\section{ABBREVIATIONS}

ADR: Adverse Drug Reaction; ICU: Intensive Care Unit; OTC: Over the Counter; FBS: Fasting Blood Sugar; INR: International Normalized Ratio; BSA: Body Surface Area.

\section{REFERENCES}

1. Kucukarslan SN, Peters M, Mlynarek M, Nafziger DA. Pharmacists on rounding teams reduce preventable adverse drug events in hospital general medicine units. Arch Intern Med. 2003;163(17):2014-8. https://doi.org/10.1001/ archinte.163.17.2014; PMid:14504113.

2. Makowsky MJ, Schindel TJ, Rosenthal M, Campbell K, Tsuyuki RT, Madill HM. Collaboration between pharmacists, physicians and nurse practitioners: a qualitative investigation of working relationships in the inpatient medical setting. J Interprof Care. 2009;23(2):169-84. https://doi. org/10.1080/13561820802602552; PMid:19234987.

3. Farsaei S, Sabzghabaee AM, Zargarzadeh AH, Amini M. Effect of pharmacist-led patient education on glycemic control of type 2 diabetics: a randomized controlled trial. J. Res Med Sci. 2011;16(1):43-9. PMid:21448382 PMCid:PMC3063424. 